\title{
Aspects of thin film deposition on granulates by physical vapor deposition
}

\author{
Andreas Eder ${ }^{1, a}$, Gerwin H.S. Schmid ${ }^{1,2}$, Harald Mahr ${ }^{1}$, and Christoph Eisenmenger-Sittner ${ }^{1}$ \\ 1 Vienna University of Technology, Institut of Solid State Physics, E-138 Wiedner Hauptstrasse 8-10, 1040 Vienna, Austria \\ 2 Austrian Institute of Technology, Energy Department, Giefinggasse 2, 1210 Vienna, Austria
}

Received 7 July 2016

Published online 22 November 2016

(C) The Author(s) 2016. This article is published with open access at Springerlink.com

\begin{abstract}
Thin film and coating technology has entered fields which may show significant deviations from classical coating applications where films are deposited on plane, sometimes large substrates. Often surfaces of small and irregularly shaped bodies have to be improved in respect to electrical, thermal or mechanical properties. Film deposition and characterization on such small substrates is not a trivial task. This specially holds for methods based on Physical Vapor Deposition (PVD) processes such as sputter deposition and its ion- and plasma assisted varieties. Due to their line of sight nature a key issue for homogenous films is efficient intermixing. If this problem is mastered, another task is the prediction and determination of the film thickness on single particles as well as on large scale ensembles thereof. In this work a mechanism capable of uniformly coating up to $1000 \mathrm{~cm}^{3}$ of granulate with particle sizes ranging from approx. $10 \mu \mathrm{m}$ to $150 \mu \mathrm{m}$ by magnetron sputtering is thoroughly described. A method for predicting the average film thickness on the particles is presented and tested for several differently shaped objects like microspheres, irregular grains of sinter powder or micro diamonds. For assessing the film thickness on single particles as well as on particle ensembles several complementary methods based on optics, X-ray analysis and gravimetry are employed. Their respective merits and limitations are discussed. Finally an outlook on adapting the described technology for surface modification by plasma based reactive and non-reactive processes is given.
\end{abstract}

\section{Introduction}

The improvement of surfaces and interfaces by coatings has gained tremendous importance during the last few decades. The progress in up-scaling coating technologies like reactive magnetron sputtering has made it possible to apply films not only to relatively small plane and smooth substrates as e.g. optical components, but also to apply high quality coatings on large substrates like window panes, solar cell panels or large sheet like components in general [1-4]. On the other hand there is also a trend to improve the mechanical, electrical, chemical or thermal properties of small, complexly shaped objects. Interfaces in modern high performance composite materials may be optimized by coating the reinforcing particles. Films with thicknesses in the range of the mean free path of conduction or heat carriers (electrons or phonons), i.e. around and below $100 \mathrm{~nm}$ at room temperature, can significantly modify the electrical and thermal characteristics of the material [5-7]. Catalytic coatings with thicknesses as low as some nm can be applied to small carrier particles thus making it possible to create catalyst materials with high surface areas using only a minimum amount of often rare

a e-mail: andreas.eder@tuwien.ac.at and expansive catalyst [8-10]. Also granulates and powders used in modern powder-metallurgical processes may require surface modifications by coatings to either enhance their sintering properties or to prevent the formation of undesired phases [11-13].

Well established techniques for the surface modification of particular matter are liquid phase processes like Electrochemistry or Sol Gel methods and gas phase processes like fluidized bed Chemical Vapor Deposition (CVD). Their undisputable advantage is the complete embedding of the particles to be coated within the material source, for instance the electrolyte or the gas which permeates the suspended particles in a fluidized bed [14-16]. Nonetheless, often the choice of coating materials is limited, for instance by the conductivity of the substrate, by the availability of electrolytic solutions or by the existence of suitable precursor gases. Physical Vapor Deposition (PVD) and especially magnetron sputtering, on the other hand, allows for basically a free choice of deposition materials. Materials can be deposited regardless of their melting point and Oxides, Nitrides or Carbides can be formed by choosing reactive processing routes [17]. The system geometry can be chosen freely, e.g. bottom up or top down, because the material sources remain in the solid state in the case of magnetron sputtering. If more than one 
source is used then also the deposition of compound coatings, gradient layers and multilayer systems becomes possible if the sources can be operated independently.

Nonetheless, the big disadvantage of PVD processes in general is their line-of-sight nature due to the large mean free path of the film forming particles, which is in the range of some $\mathrm{mm}$ to several $\mathrm{cm}$ depending on the pressure in the deposition chamber [18]. Therefore within an ensemble of particles the beam of film forming atoms or ions is mostly blocked by adjacent granules. Deposition happens only on the particles which are exposed to the vapour beam on the surface of the powder. Therefore it becomes clear that, if particles are to be coated by PVD methods in general and by magnetron sputtering in special the paramount issue for coating uniformity is efficient intermixing. There are several phenomena which have to be tackled in this respect: the change of intergranular adhesion under vacuum conditions as compared to ambient atmosphere, periodic agglomeration during deposition, charging issues due to the exposure to the magnetron plasma and the possible damage of the material to be coated by intermixing mechanisms.

Once these issues are resolved, methods to predict and to determine the film thickness on the particles are important to be developed. As concerning the prediction of the film thickness, several key factors enter the theory: the total volume of the granulate, the mean grain size, the particle shape which reflects in the trickling behaviour during deposition and the friction properties of the single particles, influences agglomeration by intergranular forces. Finally, for the determination of the film thickness on single particles as well as on particle ensembles methods known for coatings on large, plane substrates cannot readily be transferred to small, sometimes irregular particles. Therefore optical, physical analytical or gravimetric methods have to be adapted to this problem to allow for a comparison with theoretical predictions.

The above points will be tackled in systematic order within the present paper: in Section 2 the underlying principles and the basic set-up of the magnetron sputter device for coating granulates will be given. In Section 3 a theoretical approach to the prediction of the mean thickness of coatings on large ensembles of particles (up to $10^{14}$ granules) will be presented. In Section 4 the thickness of deposited material on several types of particles of different mean sizes and shapes is assessed by different complementary methods and compared to the theoretical predictions. Section 5, finally, will discuss and summarize the results. Possible upgrades of the equipment to modify the surfaces of the particles by reactive and non reactive plasmas will be presented.

\section{Design of the deposition system}

The basic features of the deposition system presented here is a moveable container which contains the granules. The container is freely positioned on a circular plate which is tilted by a given angle. If the plate is rotated, the container is lifted by small protrusions mounted on the plate until its centre of gravity wanders beyond the protrusion. Then the container is gliding along the tilted surface until it is stopped at the rim of the concussion vessel. If one assumes the mass of a single cubic particle (side length $50 \mu \mathrm{m}$, density $10 \mathrm{~g} / \mathrm{cm}^{3}$ ) to be approx. $1 \mu \mathrm{g}$, then for a lifting height of about $H=5 \mathrm{~cm}$, upon neglecting the friction between container and plate, conservation of energy yields the speed of the container $v$ to be $v=\sqrt{2 g H}=$ approx. $0.1 \mathrm{~m} / \mathrm{s}$. Considering the time for stopping at the rim of the plate to be $0.1 \mathrm{~s}$, then the inertial force exerted onto one particle by the abrupt stop can be calculated to be $F=m \cdot \Delta v / \Delta t=$ approximately $1 \mu \mathrm{N}$. Following the calculation given in reference [19] the force needed to separate one $\mathrm{nm}^{2}$ of Van der Waals bonded contact surfaces can be estimated to be about $10-20 \mathrm{nN}$ for Al with a surface tension $\gamma=1.44 \mathrm{~J} / \mathrm{m}^{2}$. Therefore the inertial force exerted on the particles should be more than sufficient to overcome the contact forces existing e.g. between the surfaces of two metal coated particles. On the other hand these forces are low as compared to the Force needed to separate for instance an area of $50 \times 50 \mu \mathrm{m}^{2}$ at a yield strength of $100 \mathrm{MPa}$, which amounts to $0.25 \mathrm{~N}$. So it can be expected that damage to the particles is unlikely, even if crack initiation is not considered here.

Smaller versions of this mechanism as well as the reasons for the choice of a very specific container geometry are described in detail in references [20,21]. In the following an upscaled set-up based on the same principle of a rotating, periodically concussed container which is capable of coating $10^{3} \mathrm{~cm}^{3}$ of granulate (which, if one again takes particles of approx. $50 \mu \mathrm{m}$ linear extension amounts to about $10^{12}$ particles with an approximate surface of $72 \mathrm{~m}^{2}$ ) will be presented. Also modifications of the container geometry and the optimization of the position of the sputter sources in respect to the exposed surface of the powder to achieve maximum deposition rates will be discussed.

Based on the mentioned device of Schmid a new upscaled version has been developed replacing and optimizing the main parts such as the sputter sources and the concussion vessel itself. Preliminary feasibility studies in a 3D-CAD model (Fig. 1) helped dimensioning the new system. Having a single big container offers a better ratio of loading capacity to volume compared to any other system, for example three medium upscaled bowls. To handle the high load of a big coating vessel and the resulting forces, the mounting of the rotation mechanism of the original system consisting of small coating vessels has been replaced with a solid mounting driven by steel gears. It can freely be tilted between $\alpha=28^{\circ}$ and $45^{\circ}$. Varying the angle of the concussion mechanism results in different forces exerted onto the particles and also in different coating rates as the distance between target and substrate decreases the lower the angle $\alpha$ gets. Experiments have shown that the coating rate is nearly doubled when operated at $\alpha=28^{\circ}$ compared to $\alpha=45^{\circ}$. The concussion plate, representing the biggest rotating part has been designed to maximize the vessel size even further, consisting of a flat round plate with bolts for lifting 


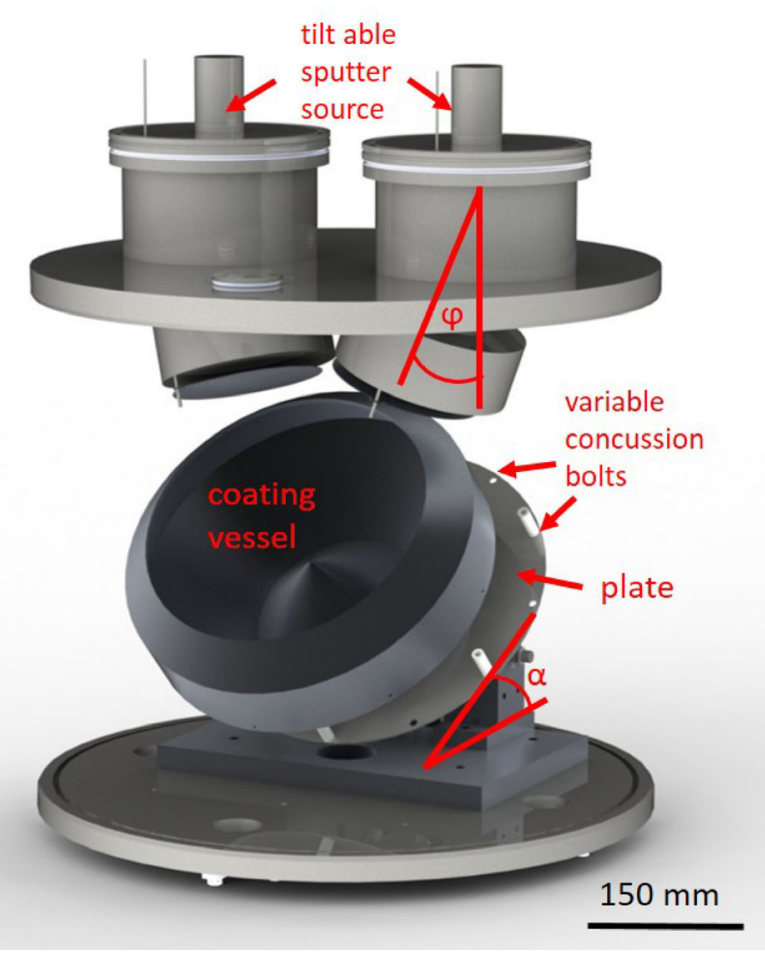

Fig. 1. 3D-model of the upscaled sputter device showing the main parts of the concussion mechanism.

the bowl perpendicular to the plate surface, arranged circular at the outer most edge. The lifting height can now be adjusted by varying the amount of bolts.

The maximum size of the bowl is only limited by the dimensions of the vacuum chamber (the cylindrical chamber used here has a diameter of $45 \mathrm{~cm}$ and height of $40 \mathrm{~cm}$ ) as well as the sputter sources extended into the chamber. As the bowl slides freely on the concussion plate, the additional space required for the movement also needs to be considered and the maximum diameter of the bowl was found to be $300 \mathrm{~mm}$. Particle ejection from the vessel, once a problem when working with small containers, can be completely avoided by adding a rim tilted $45^{\circ}$ inwards to the center of the bowl resulting in a geometry similar to a common concrete mixer. To limit the shadowing effect of the rim which would result in a decreased deposition rate, two tiltable 4 " sputter sources $\left(\varphi=0^{\circ}\right.$ to $\left.20^{\circ}\right)$ are installed in the top flange allowing to direct the vapor beam directly towards the substrate.

To align the sources and obtain maximum coating rates, a transparent flexible substrate (Black/White Laser Transparency Film, Q-Connect) has been coated at the two tilt extrema of the concussion plate which are $28^{\circ}$ and $45^{\circ}$. The sputter rate distribution of the sputter sources was determined by measuring the light intensities of an image taken with an optical transmission scanner as given in Figure 2a. The shape of the coated foil was chosen to be the surface of an ideal fluid at a filling level of one liter in the coating vessel as shown in Figure $2 \mathrm{~b}$.

A very large, nearly homogeneous area with similar intensity distribution and clearly visible maxima of the two sputter sources is observable. It was found that this is independent of the tilt angle $\alpha$ of the concussion plate. The outer most area with a length of about $2 \mathrm{~cm}$ and an area of about $13 \%$ of the total exposed surface shows a clearly visible gradient also marked in Figure 2a caused by the rim obstructing the particle beam. The covered area even increases to a maximum of $35 \%$ at a lower tilting angle of $\alpha=28^{\circ}$ as the rim was optimized for operation at $45^{\circ}$, however the total rate increases as already mentioned due to the decreased distance.

\section{Prediction of the film thickness on granulates for line-of-sight processes}

As commonly known, the smaller a particle is, the higher is the surface to volume ratio, resulting in long deposition times for large total surfaces. To keep the deposition times as low as possible to deposit the desired film thickness, the need of a reliable film thickness prediction is evident. In general the deposition rate $R$ on plane substrates is the relation of deposited film thickness $x$ in a certain amount of time $t$ as shown in equation (1) and can be easily determined in an experiment

$$
R=\frac{x}{t}
$$

Taking into account that the diameter of granulates coatable with this device ranges from $150 \mu \mathrm{m}$ to $10 \mu \mathrm{m}$ the total surface $A_{t o t}$ of one litre spherical granulate ranges from $24 \mathrm{~m}^{2}$ to $360 \mathrm{~m}^{2}$, which is quite large compared to the area $A_{\text {expo }}$ exposed to the vapour beam (a few hundred $\mathrm{cm}^{2}$ ), thus limiting the effective deposition rate $R_{e}$ on a single particle, leading to equation (2). In general, $A_{\text {expo }}$ is depending on the coating geometry, and in this special case is mainly influenced by the size of the container, the angle between sputter source and container, the trickling behaviour and the vapour beam distribution

$$
R_{e}=\frac{x A_{t o t}}{t A_{\text {expo }}} .
$$

As a first approach the exposed area of the substrate is approximated by a function depending on the volume of substrate filled in the coating container while standing still. The granular materials are assumed to have the behaviour of a fluid, which is almost the case under ambient conditions. This means that the surface of the granulate is represented by a horizontal plane. The intersection of the tilted container with this plane yields the shape of the exposed surface. A semi-empirical study with a CAD Software at two tilting angles (Fig. 3) led to the following equation (3) with the fitting constants $A_{x}, t_{x}$ and $y_{0}$ and the variable volume of the substrate $V_{\text {Substrate }}$

$$
\begin{aligned}
A_{\text {expo }}= & y_{0}+A_{1}\left(1-\exp \left(\frac{-V_{\text {Substrate }}}{t_{1}}\right)\right) \\
& +A_{2}\left(1-\exp \left(\frac{-V_{\text {Substrate }}}{t_{2}}\right)\right) .
\end{aligned}
$$




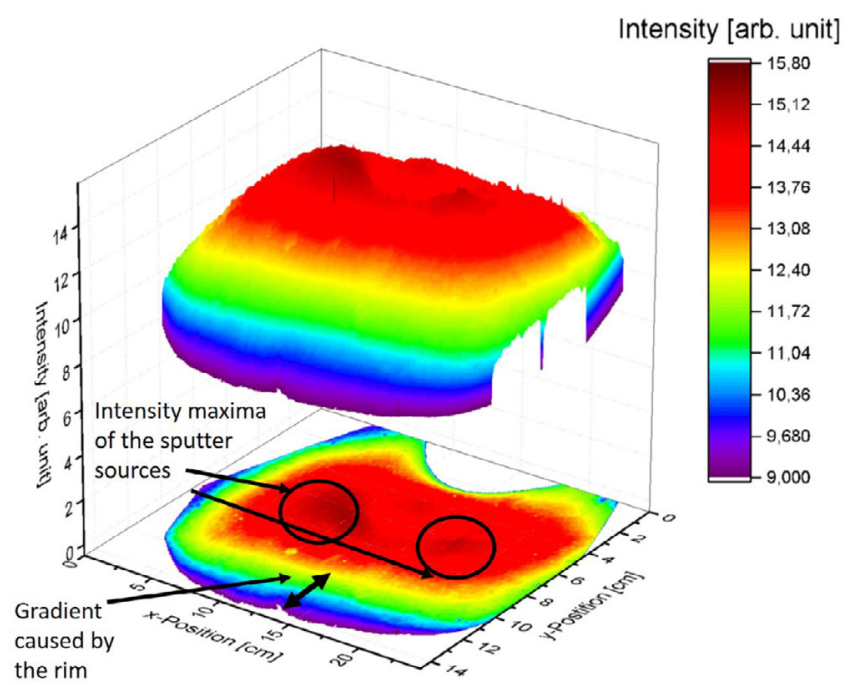

(a)

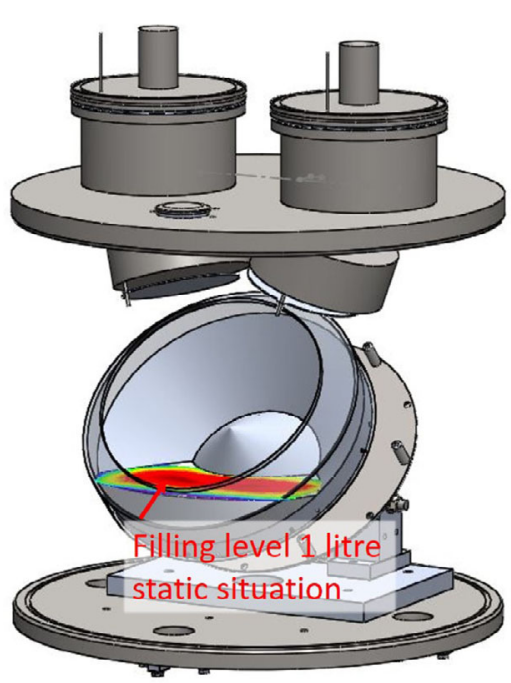

(b)

Fig. 2. (a) Distribution of intensity of sputtered particles at $45^{\circ}$ tilted concussion plate. (b) Filling level of 1 litre ideal granular substrate in the static situation.

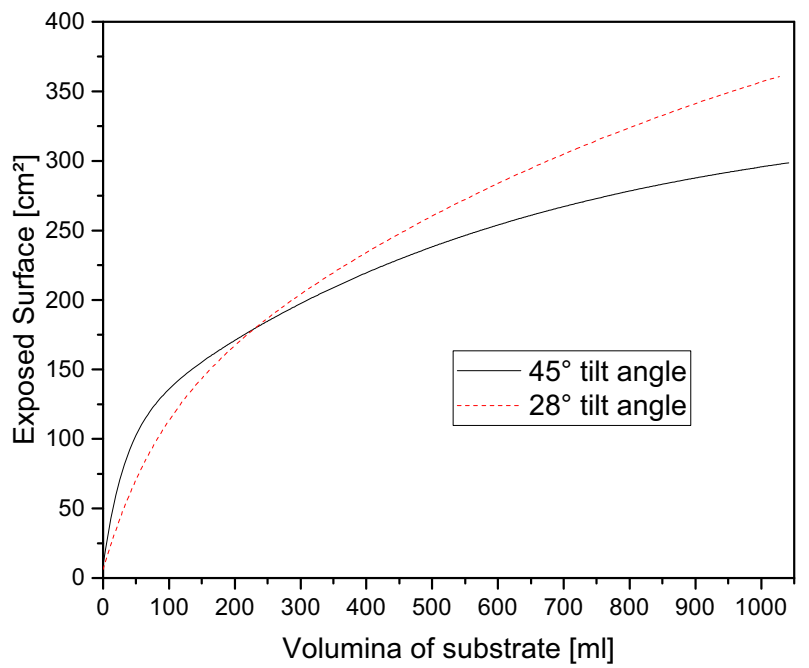

Fig. 3. Exposed area in relation to coated volume dependent on the tilting angle of the large coating vessel.

In fact, this approximation is not quite accurate since the trickling behaviour of the granulate degrades in vacuum. It partly sticks to the bowl during operation under vacuum, thus increasing the exposed area. In addition the exposed area is not parallel to the top flange anymore due the effective viscosity of the granulate. Therefore it is closer to one sputter source at one side. Thus a single intensity maximum develops as displayed in Figures $4 \mathrm{a}$ and $4 \mathrm{~b}$.

According to the Hertz-Knudsen-law the deposition rate follows a $1 / r^{2}$ relation where $r$ is the distance between target and substrate. Comparing the distances of the substrate surface in the static case with the dynamic case a reduction from $20 \mathrm{~cm}$ to $15 \mathrm{~cm}$ can be assumed (peak maxima to target) resulting in a doubled deposition rate. Experiments with different mean particle sizes ranging from $20 \mu \mathrm{m}$ to $130 \mu \mathrm{m}$ and different shapes such as spheres or diamonds with a shape close to truncated octahedra have shown much more complex granulate distributions and so an empirical correction factor of the exposed area of 1.5 to 3 is needed to obtain accurate results. This factor is mainly related to the grain size and shape of the granulate, and essentially independent of the target material, indicating that the trickling behaviour is the main reason for the correction needed. Finding an analytic expression describing the trickling under vacuum conditions is therefore an interesting task needed to be discussed in detail in future work.

The total surface area $A_{t o t}$ is the surface of one sphere $A_{\text {Sphere }}$ times the total number of spheres $n$. $n$ can be expressed by the packing factor $f$ and the result for $A_{t o t}$ is shown in equation (4). $V_{\text {Substrate }}$ is the volume one wants to coat and $V_{\text {Sphere }}$ is the volume of one Sphere of the granulate

$$
\begin{aligned}
A_{\text {tot }} & =n A_{\text {Sphere }} \\
& =\frac{f V_{\text {Substrate }}}{V_{\text {Sphere }}} A_{\text {Sphere }} \\
& =\frac{3 f V_{\text {Substrate }} f_{S}}{r_{S}} .
\end{aligned}
$$

In addition a factor $f_{S}$ has to be considered in equation (4) which corresponds to the shape of the particles with a mean radius $r_{S}$. For spheres with smooth sufaces it is 1 (for example glass microspheres) and needs to be adjusted the more edged or rougher the surface gets. For diamonds, which can be geometrically approximated by truncated octahedra, the exposed area of a single grain on the average is limited to about $1 / 3$ of the total particle surface. As every part of the surface needs to be exposed equally to the vapour beam the effective total surface needs to be considered and results in a form factor of $f_{S}=1.5$. Elongated grains such as Magnesium dopedPartially Stabilized Zirconium-oxide (Mg-PSZ) particles are approximated with a cylindrical shape, with a height 


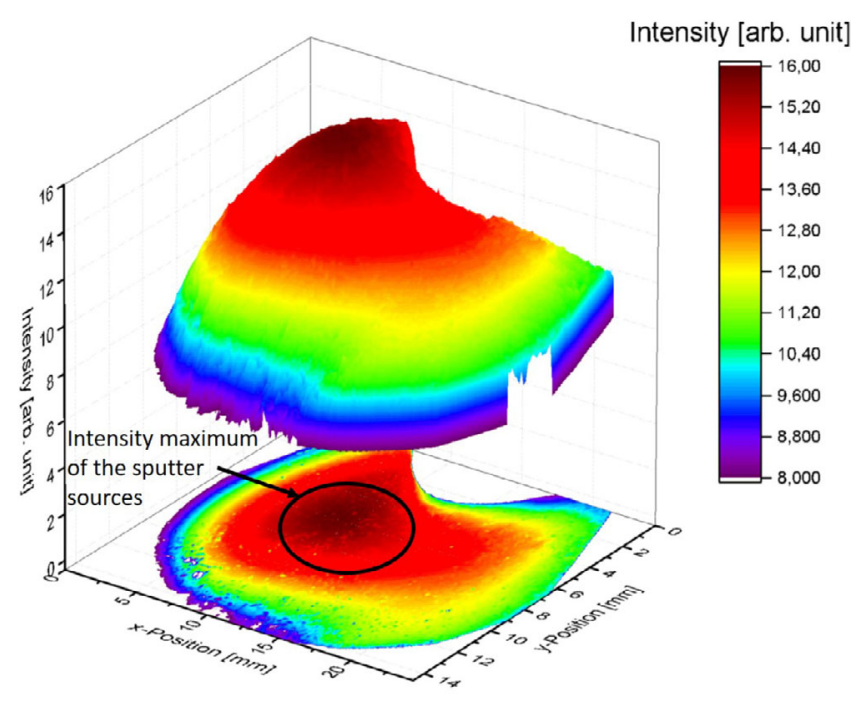

(a)

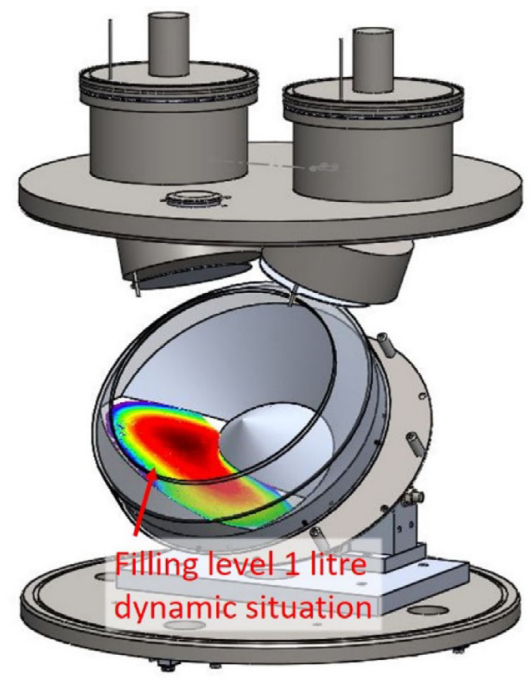

(b)

Fig. 4. (a) Distribution of intensity of sputtered particles at $45^{\circ}$ tilted concussion plate dynamic situation. (b) Filling level of 1 litre granular substrate in a dynamic situation.

Table 1. Coating times for different substrates at two tilting angles of the bowl at constant rates, calculated with the prediction model.

\begin{tabular}{cccc}
\hline & $r_{S}[\mu \mathrm{m}]$ & \multicolumn{2}{c}{$t[\mathrm{~h}]$} \\
Substrate & & $\alpha=28^{\circ}$ & $\alpha=45^{\circ}$ \\
\hline HGM & 19.03 & 14.2 & 23.4 \\
Mg-PSZ & 20.59 & 19.7 & 32.4 \\
Diamonds & 135 & 3 & 4.9 \\
\hline
\end{tabular}

to radius ratio of $h=3 r$, resulting in the same ratio of total surface to effective exposed area as diamond grains.

Combining equations (2) and (4) leads to equation (5) where either the necessary sputter time $t$ or the film thickness $x$ can be predicted

$$
x=\frac{r_{S} R t A_{\text {expo }}}{3 V_{\text {Substrate }} f f_{S}} .
$$

This equation uses well known input parameters besides the semi empiric value $A_{\text {expo }}$, which needs to be determined more precisely in the future. The deposition rate at certain sputtering powers and vessel geometries can be easily determined in an experiment, the amount of substrate to be coated can be measured and the packing factor is often given by the manufacturer of granular materials. To show the validity of equation (5), the theoretical prediction will always be compared to the experimental results in the next sections. In conclusion the derived expressions can be used to predict e.g. the time needed to achieve a given Copper film thickness $(x=50 \mathrm{~nm})$ as done in Table 1 for different substrates (each 1 litre) and angles at constant coating rates.

\section{Film thickness measurement methods}

To assess the film thickness on a specific granulate multiple methods are used, which will be discussed in detail and then will be compared. Since every evaluation method is only valid within some restrictions a substrate where all methods can be used will be discussed, i.e. Hollow Glass Microspheres (HGMs) S38 manufactured by 3M, commercially available in large quantities. The target material mainly used will be copper which is a good compromise in the matter of cost, sputter yield, target cooling and maximum power at which targets can be operated. The samples presented in this chapter are prepared using two 4-inch $\mathrm{Cu}$-targets each operated at a $\mathrm{DC}$ power of $1 \mathrm{~kW}(U=500 \mathrm{~V}, I=2 \mathrm{~A})$ at an Argon pressure of 0.4 Pascal. At this given gas pressure and due to the high distance between substrate and target $(\sim 15-20 \mathrm{~cm})$ particle - plasma interactions as e.g. particle charging or a dusty plasma $[22,23]$ are unlikely. Furthermore there is no interaction between the plasma sources in simultaneous operation observed, as the Voltage and Current is the same when operated solo.

\subsection{Gravimetric measurement}

A very simple and effective method to determine the film thickness $x$ is to measure the increasing mass $m$ of the substrate and calculate the film thickness according to equation (6)

$$
x=r_{S}\left(\sqrt[3]{\left(\frac{m_{\text {film }}}{\rho_{\text {film }} f f_{S} V_{\text {Substrate }}}\right)+1}-1\right) .
$$

This equation can be derived by distributing the measureable mass increase $m_{f i l m}=V_{\text {film }} \rho_{\text {film }}$ equally to all $n$ 


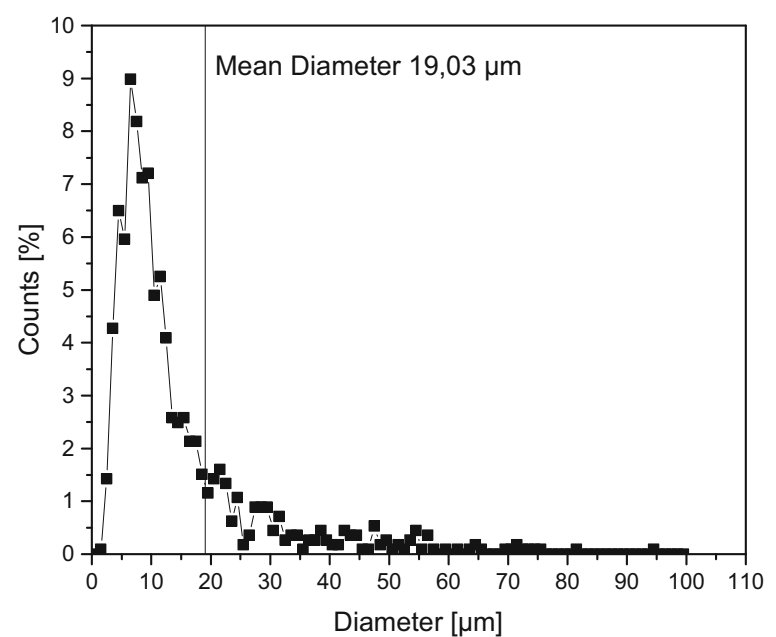

(a)

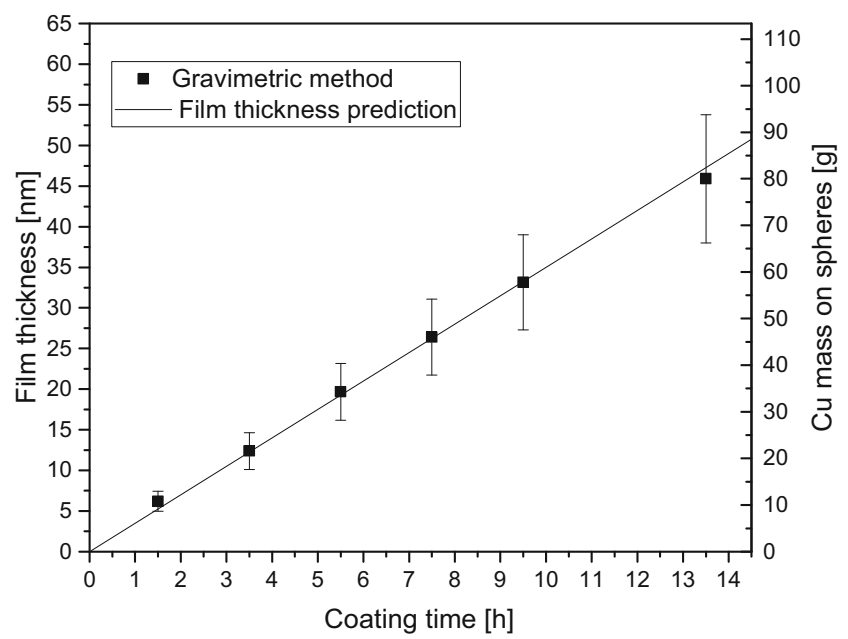

(b)

Fig. 5. (a) Particle size distribution of HGMs with marked surface weighted mean diameter. (b) Increasing mass and calculated film thickness of copper coated HGMs.

spheres with a volume of $V_{\text {Sphere }}$ with a mean sphere radius $r_{S}$. By using the packing factor $f=0.6 \pm 0.05$ (as already used in Eq. (4)) the amount of spheres $n$ contained in the coated total volume $V_{\text {Substrate }}$ can be calculated. It is about $10^{19}$ spheres per litre (mean radius $r_{S} \sim 19 \mu \mathrm{m}$ ). As already discussed earlier, $f_{S}$ is the form factor of the granular material and in case of spheres as shown here $f_{S}=1$. Since the results represent a mean thickness they give no information on the thickness homogeneity. In addition the density of the sputtered film, in case of a single material film, is approximated with the bulk density of the material sputtered. Ejected particles would also influence the results since the total surface gets reduced during coating. However, this effect is not present in the current sputter device. The detailed measurement procedure is as follows: (i) weigh empty container; (ii) weigh filled container; (iii) weigh coated and filled container; (iv) weigh empty but still coated container.

The largest influence in calculating the film thickness, of course, is the mean particle radius $r_{S}$. Getting access to this parameter is therefore an important task which can be accomplished by measuring the diameter of the particles. As an example the diameter of about $10^{3}$ HGMs has been measured by image analysis of several SEM images. The particle size distribution can be seen in Figure 5a. Another possible method which delivers representative diameters of arbitrarily shaped particles would be laser diffraction particle size analysis (for instance: Sympatec, HELOS/BR [24]).

Due to the fact that only the surface is coated it is intuitive to weigh the particle diameter in respect to the mean surface which is done by first calculating the surface of each particle $A_{i}$ with a measured diameter $d_{i}$, average this data to a mean surface $A_{\text {mean }}$ of a representative sphere and afterwards recalculate the surface weighed mean surface weighed diameter $d_{\text {mean }}(A)$ as seen in equation (7). A similar approach can be done with the volume (volume weighed)

$$
A_{\text {mean }}=\frac{1}{n} \sum_{i}^{n} A_{i} \rightarrow d_{\text {mean }}(A)=\sqrt{\frac{\sum_{i}^{n} d_{i}^{2} \pi}{\pi n}} .
$$

The size distribution of HMGs would result in diameters of $14.21 \mu \mathrm{m}, 19.03 \mu \mathrm{m}$ and $23.71 \mu \mathrm{m}$, not weighted, surface weighted, and volumetric weighted mean diameters respectively.

Keeping the sputter parameters influencing the deposition rate (e.g. power, distance between substrate and target) constant and just increasing the deposition time, a linear mass increase is observed (Fig. 5b), the film thickness can be calculated and the results are coinciding well with the prediction model.

\subsection{Optical microscope}

Measuring the film thickness for transparent substrates and absorbing films based on Beer-Lamberts law (Eq. (8)) is a common method although not often used on small particles because additional precautions have to be met which will be discussed here. The transmission $T$ of a monochromatic EM-wave through an absorbing medium (extinction coefficient $\alpha$ ) is given by:

$$
T=\frac{I}{I_{0}}=e^{-\alpha d}
$$

The measurement in general is based on comparing intensities of a light beam before $\left(I_{0}\right)$ and after passing a certain substrate $(I)$ in dependence on the thickness $d$ and the extinction coefficient $\alpha$. As the material the granulate is coated with is well-known, the extinction coefficient is also well defined. The intensities $I_{0}$ and $I$ can be determined by evaluating the RGB channels of an image taken 


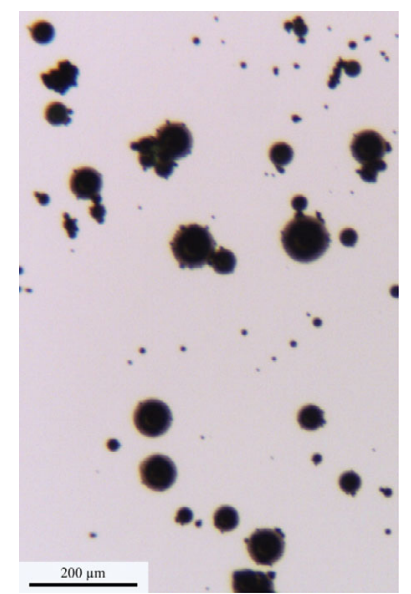

(a)

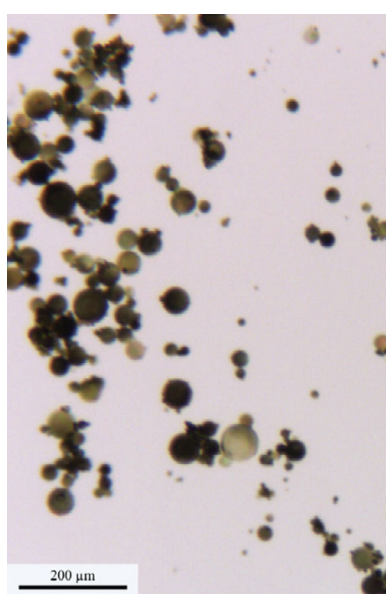

(b)

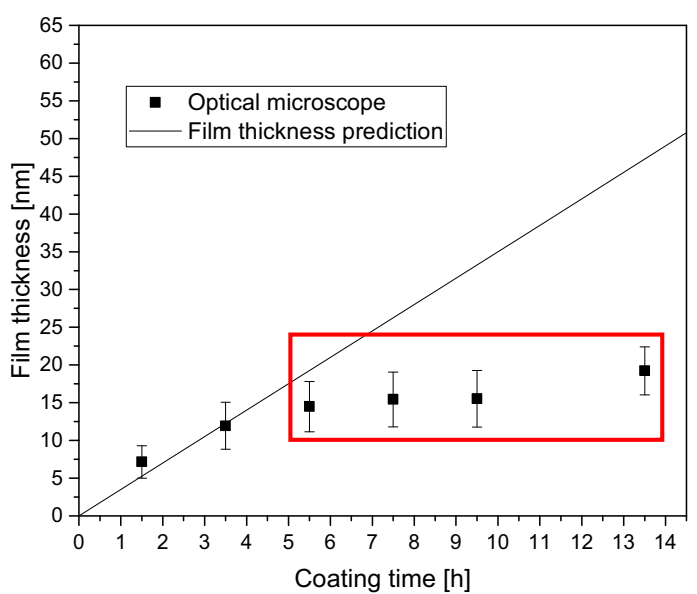

(c)

Fig. 6. (a) Homogeneous films on HGMs picture using an optical microscope. (b) Inhomogeneous films on HGMs. (c) Evaluation of the film thickness using the extinction coefficient at $\lambda_{\text {red }}=580, \lambda_{\text {green }}=523, \lambda_{\text {blue }}=477$; thick films cause a saturation as the light can't penetrate the film any more (red frame).

with a common optical microscope or a high resolution scanner. To get proper images for the film thickness evaluation, a single layer of granulate needs to be prepared and agglomerations should be avoided. By subtracting the background and using a proper object identification software, single objects can be isolated and the intensities can be extracted which then can be used to calculate a film thickness according to equation (8). Typical pictures of homogeneous and clearly visible inhomogeneous copper coated spheres can be seen in Figures 6a and 6b. By taking several pictures the amount of spheres measured can easily range up to 1000 and therefore provide good statistics. In Figure 6c one can see well fitting measurement values compared to the theoretical prediction at coating times up to $3.5 \mathrm{~h}$ and a film thickness of about $13 \mathrm{~nm}$.

The transmission intensity at a certain wave length is dependent on the film thickness and only amounts to approximately $5 \%$ at a thickness of $d=50 \mathrm{~nm}$ at a wavelength of $\lambda=523 \mathrm{~nm}$ [25] for $\mathrm{Cu}$. The CCD chip of the microscope uses the following wavelength: $\lambda_{\text {red }}=580$, $\lambda_{\text {green }}=523, \lambda_{\text {blue }}=477$. As we have coated spheres and the light needs to pass the film twice, the theoretical limit of a still measurable film thickness would be about $25 \mathrm{~nm}$ in case the ccd chip of the camera or the scanner uses this given wavelength. Experiments have shown a saturation (as marked in Fig. 6c, red frame) at a maximum film thickness of about $15-20 \mathrm{~nm}$ copper film thickness which corresponds to the theoretical limit quite well.

\subsection{Scanning electron microscope}

Using an imaging technique has the advantage of obtaining visual information on the films and the film thickness itself. A Scanning Electron Microscope (SEM) allows much higher resolutions compared to a common optical microscope and detailed analysis of the HGMs surface as seen in Figure 7a. The copper coated spheres provide the necessary electrical conductivity for SEM imaging and the low contrast difference and smooth looking surface indicates a homogeneous film thickness distribution. The small bright dots, enlarged in the inset of Figure 7a represent the anti caking agent (silicid acid) applied by the sphere manufacturer to supress agglomeration under atmospheric conditions. Breaking the coated HGMs offers easy access to the cross section and requires no further preparation as needed for example for a TEM lamella. While breaking the spheres no film delamination is observed and it even seems that the anti caking agent (Fig. 7b) adhesion is supported by the coating as no peeling occurs. In general the film adhesion on a substrate of choice can be influenced by a plasma cleaning pretreatment which is planned to be implemented in the coating device and discussed in the outlook of this paper. The only requirement to assess the film thickness is a broken edge perpendicular to the incoming electron beam. In Figure $7 \mathrm{~b}$ once again the very homogeneous film can be observed, the thickness varying at the sub nanometer scale. Due to the small sample size of analysed spheres and the need of a minimum film thickness of about $25 \mathrm{~nm}$ for reasonable images the SEM can only be used as a spot check of the results acquired with the measurement methods presented before.

\subsection{EDX-penetration depth}

Electrons with different energies have a certain penetration depth in different materials depending on their atomic number $Z$. Since the excitation volume of an electron beam of a SEM capable of Energy Dispersive X-ray analysis (EDX) is dependent on the penetration depth, one can obtain a depth profile of the chemical composition. By increasing the film thickness the electron beam is getting more and more absorbed in the layer which results in a decreasing signal of the substrate and an increasing signal of the film. Complete penetration of the layer 


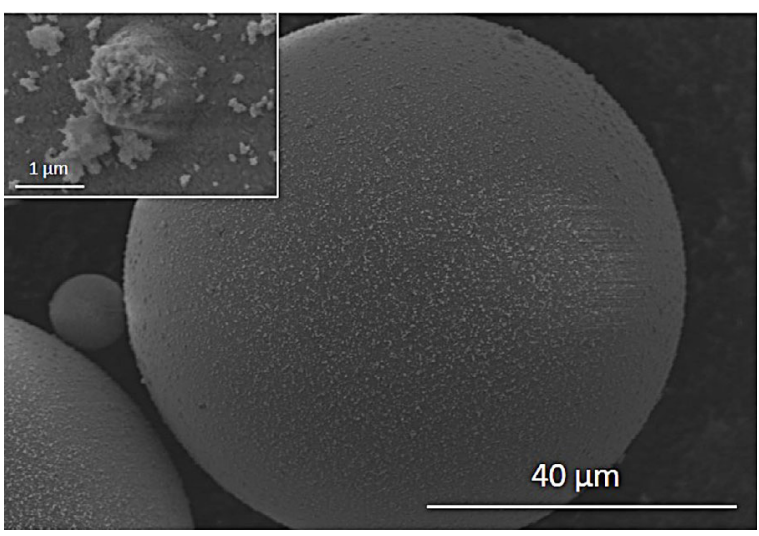

(a)

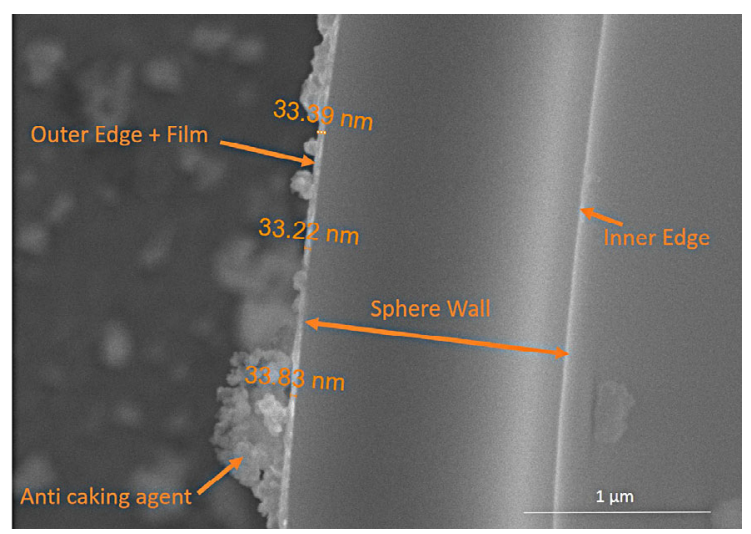

(b)

Fig. 7. (a) Surface of copper coated HGMs with a homogeneous layer; inset: anti caking agent. (b) Cross section of a $7.5 \mathrm{~h}$ copper coated HGMs.
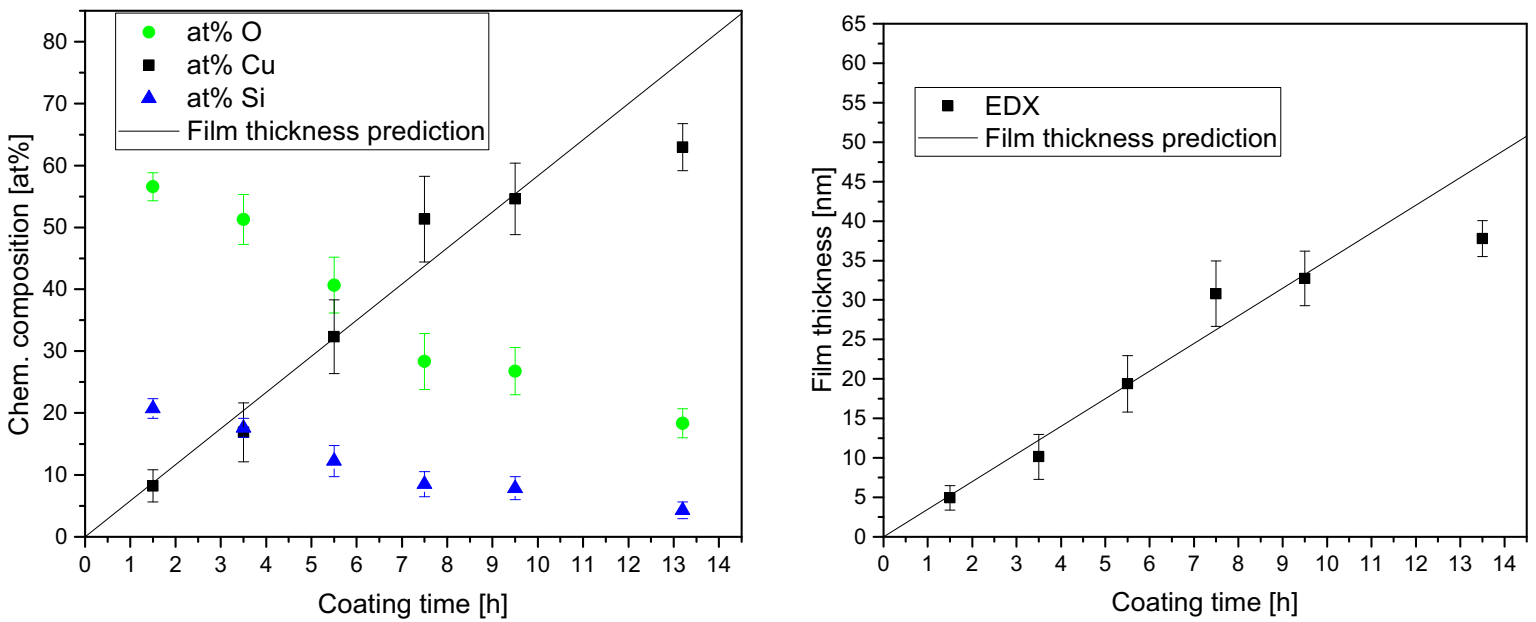

Fig. 8. (a) Chemical composition measured with $5 \mathrm{kV}$ acceleration voltage of different coating times. (b) Film thickness calculated using chemical composition and penetration depth.

and the substrate underneath would result in incorrect quantification of the chemical composition. Since very thin layers are evaluated and the HGMs wall is just about $1 \mu \mathrm{m}$ thick, it is necessary to operate at a very low electron acceleration voltage $U_{a c c}$ which, however, is still high enough to excite the observed elements. In Figure 8a one can see the linear increase of the atom percentage of copper at increasing coating time while Silicon and Oxygen signals are decreasing (measurements taken at $U_{a c c}=5 \mathrm{kV}$ ). The more than doubled Oxygen content compared to Silicon results from the composition of the HGMs which are made of borosilicate glass and therefore contain besides mainly $\mathrm{SiO}_{2}$ also other oxides like $\mathrm{B}_{2} \mathrm{O}_{3}$ or $\mathrm{Al}_{2} \mathrm{O}_{3}$. According to Win X-Ray [26] simulations the theoretical maximum penetration depth of electrons with an energy of $5 \mathrm{keV}$ is about $100 \mathrm{~nm}$ and $400 \mathrm{~nm}$ in $\mathrm{Cu}$ and $\mathrm{SiO}_{2}$, respectively. The electron beam should therefore not totally penetrate the sphere shell. Nevertheless, fitting the Si concentration to the point where the composition is $0 \mathrm{at} \% \mathrm{Si}$ would result in $15.55 \mathrm{~h}$ coating time and a $\mathrm{Cu}$-film thickness of $54 \mathrm{~nm}$. Measuring thicker films at $5 \mathrm{kV}$ is therefore impossible as a relation of $0 \% \mathrm{SiO}_{2}$ to $100 \% \mathrm{Cu}$ would suggest the presence of bulk copper. To avoid incorrect results, the EDX signal was acquired at areas of about $50 \%$ of the sphere diameter to ensure that pores or cracks in the film can be neglected, as the electron beam would penetrate these defects much more than a homogeneous dense film.

By fitting the EDX data with the film thickness assessed from cross sections, and comparing it with the prediction model and the other film thickness evaluation methods discussed in this paper a relation of 1 at\% $0.6 \mathrm{~nm}$ can be established. As already mentioned this calibration factor found is valid for copper films as long as the silicon substrate is monitored independently of the deposition parameters of the film on HGMs. The calculated film thickness is displayed in Figure 8b.

\section{Discussion}

Thin films ranging from metals to oxides or nitrides can be applied homogeneously on quantities of up to one litre 
of granular material e.g. hollow glass microspheres, diamonds or Magnesium doped- Partially Stabilized Zirconia. The coating vessel and agglomeration suppression mechanism necessary to sustain trickling are an upscaled version of the special coating geometry developed by Schmid [20,21]. By rearranging the sputter sources and tilting them towards the bowl it was possible to apply a rim to the coating vessel totally supressing particle ejection while shading effects are kept to a minimum. A semi empiric model to predict the film thickness has been developed, valid for arbitrary shaped particles and sizes. Factors influencing the calculation such as the vapour beam exposed surface area or trickling behaviour have been approximated analytically but still need further improvement. The thickness calculations have shown their good accuracy as compared with different methods of film thickness determination:

- Gravimetric: measuring the weight gain of coated granulate and distributing it to all the particles equally, offers the possibility to calculate the film thickness with high precision but granting no information on the homogeneity.

- Optical: optical film thickness determination, providing good statistics on large ensembles of granulates, is limited to transparent substrates. Reliable results comprising average thickness, global film thickness distributions and film homogeneity are obtained, as long as the method is applicable.

- SEM/EDX: relating the chemical composition obtained from EDX spectra based on the different grade of absorption of the electron beam to a certain film thickness offers an indirect way to assess the film thickness. Nevertheless, statistics are rather small and only spot checks of the film thickness can be done by measuring the film thickness of cross sections.

Combining the introduced measurement methods for copper coated hollow glass microspheres as displayed in Figure 9 provides a consistent picture. The described methods yield the same results where thickness measurement is possible and cover different complementary thickness ranges.

\section{Conclusion and outlook}

In conclusion a system has been presented which allows for coating approximately 1 litre of granulate irrespective of particle shape and size. A method for the prediction of the film thickness on the particles has been developed and was tested by several complementary methods of thickness determination. The coating system is capable of implementing a plasma close to the granular substrate thus offering additional possibilities of surface treatment and modification.

To get further information on the film structure, and even assess the film thickness, the electrical conductivity of metal coatings of coated granulates can be mea-

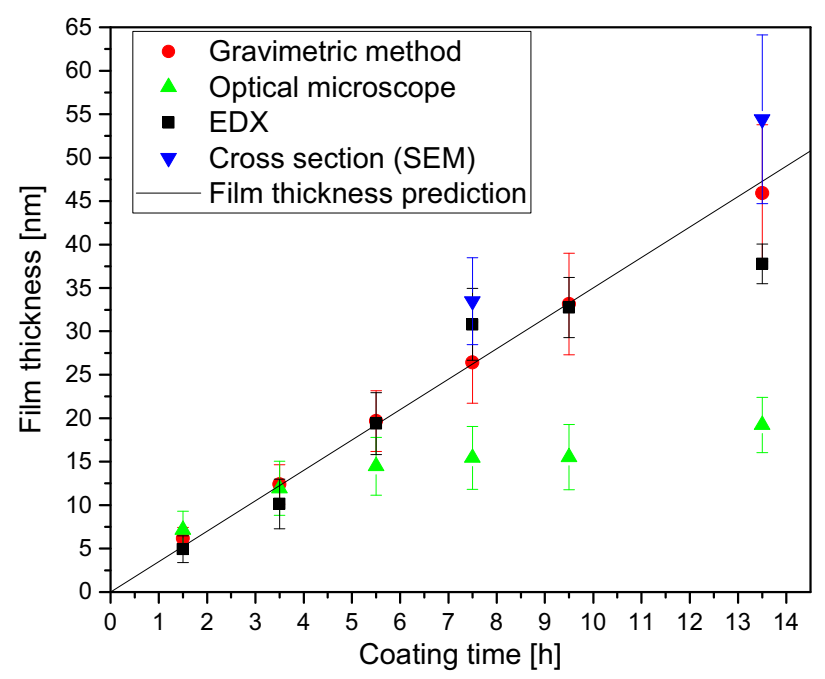

Fig. 9. Film thickness prediction and evaluation methods compared.

sured. For that a powder needs a compressing force to be connective. A device will be built consisting of two copper cylinders which compress the powder with a tuneable force, similar to references $[27,28]$. Another task of future work will be the surface pretreatment of granular materials by plasma etching, representing a common method often implemented in sputter processes. The coating vessel has therefore been already electrically insulated and set on high negative potential to localize the plasma within the bowl. To intensify the plasma a magnetic system, similar to a magnetron has been developed to fit into the central cone of the coating vessel. Meander shaped magnetic loops have been placed on an Iron cone as seen in Figure 10a. The magnetic orientation is perpendicular inwards and outwards, coloured red and green respectively. Igniting the plasma results in a starfish formed racetrack (Fig. 10b) with six loops located inside of the coating vessel.

Rotating the coating vessel also rotates the magnetic field as well as the granular substrate and allows the ion bombardment of the granulate surface, cleaning it, changing its morphology or chemically activating it if reactive gasses are introduced. However, detailed experiments need to be performed to allow for a qualitative and quantitative analysis of the efficiency of this cleaning process. Up to now only non ferromagnetic particles have been coated and therefore the influence of the magnetic field on the trickling behaviour needs to be determined in future experiments.

This work is supported by the Austrian "Fonds zur Förderung der Wissenschaftlichen Forschung" (FWF) under Grant Nr.: TRP-281-N20. SEM Investigations were carried out using facilities at the University Service Centre for Transmission Electron Microscopy (USTEM), Vienna University of Technology, Austria. 

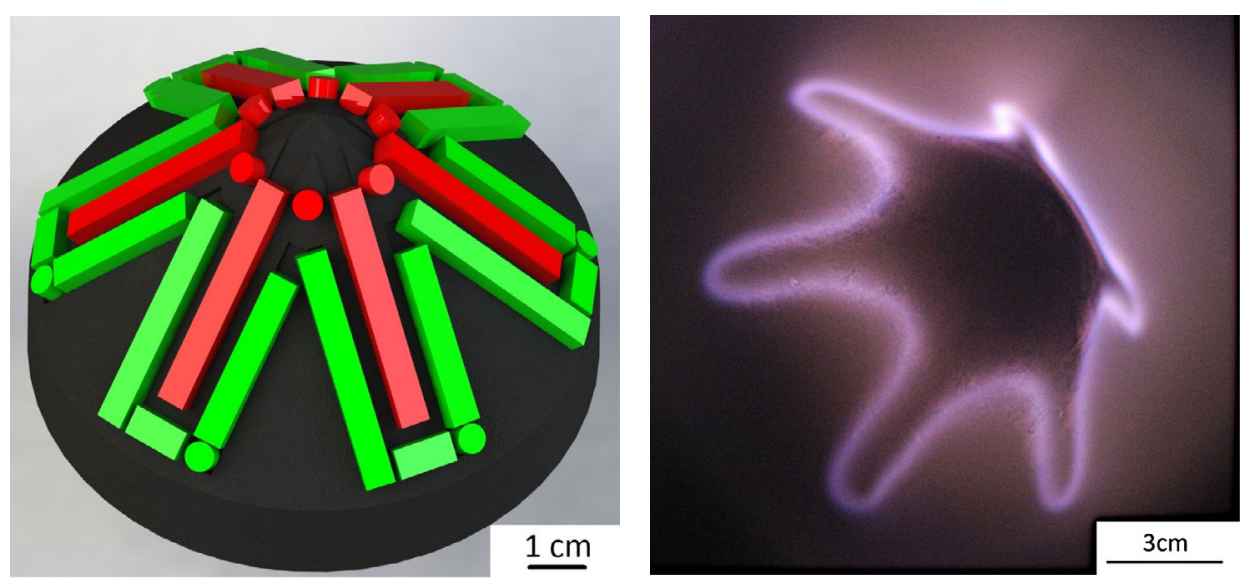

Fig. 10. (a) Magnetic system for plasma etching of granular materials. (b) Ignited plasma - starfish shaped racetracks.

\section{References}

1. S. Schiller, V. Kirchhoff, N. Schiller, H. Morgner, Surf. Coat. Technol. 125, 354 (2000)

2. N. Selvakumar, H.C. Barshilia, Solar Energy Mater. Solar Cells 98, 1 (2012)

3. C.G. Granqvist, Solar Energy Mater. Solar Cells 99, 166 (2012)

4. M. Kamalisarvestani, R. Saidur, S. Mekhilef, F.S. Javadi, Renew. Sust. Energ. Rev. 26, 353 (2013)

5. T. Köck, A. Brendel, H. Bolt, J. Nucl. Mater. 362, 197 (2007)

6. S. Scholz, S. Kaskel, J. Colloid Interf. Sci. 323, 84 (2008)

7. Th. Schubert, B. Trindade, T. Weißgärber, B. Kieback, Mater. Sci. Eng. A 475, 39 (2008)

8. Lu Wu, Haijiao Zhang, Minghong Wu, Yufang Zhong, Xingwen Liu, Zheng Jiao, Microporous and Mesoporous Mater. 228, 318 (2016)

9. L.H. Tamborini, M.E. Casco, M.P. Militello, J. SilvestreAlbero, C.A. Barbero, D.F. Acevedo, Fuel Process. Technol. 149, 209 (2016)

10. A.E. Alvarez, A.N. Gravina, J.M. Sieben, P.V. Messina, M.M.E. Duarte, Mater. Sci. Eng. B 211, 26 (2016)

11. Kuan-Wen Wang, Shu-Ru Chung, Ling-Yun Jang, Jyh-Fu Lee, Tsong-Pyng Perng, J. Alloys Compd. 415, 62 (2006)

12. Chang-Hoon Kim, Kum-Jin Park, Yeo-Joo Yoon, DongSook Sinn, Young-Tae Kim, Kang-Heon Hur, J. Eur. Ceramic Soc. 28, 2589 (2008)

13. Jae-Hoon Ahn, Jin-Kyu Lee, Dong-Woo Kim, Young-Soo Jung, Gil-Pyo Kim, Sung-Hyeon Baeck, Mater. Res. Bull. 43, 2266 (2008)

14. R.N. d'Alnoncourt, M. Becker, J. Sekulic, R.A. Fischer, M. Muhler, Surf. Coat. Technol. 201, 9035 (2007)

15. Jun Liu, A.T. Harris, Chem. Eng. Sci. 64, 1511 (2009)

16. W.O. Filtvedt, A. Holt, P.A. Ramachandran, M.C. Melaaen, Solar Energy Mater. Solar Cells 107, 188 (2012)
17. Reactive Sputter Deposition, edited by Diederik Depla, Stijn Mahieu (Springer Berlin Heidelberg, 2008)

18. R.F. Bunshah et al., Deposition Technologies for Films and Coatings - Developments and Applications (Noyes Publications, Park Ridge, New Jersey, 1982)

19. V.L. Popov, Kontaktmechanik und Reibung (Springer Heidelberg, Dordrecht, London, 2010), Vol. 2

20. G. Schmid, C. Eisenmenger-Sittner, J. Hell, M. Horkel, M. Keding, H. Mahr, Surf. Coat. Technol. 205, 1929 (2010)

21. G.H.S. Schmid, C. Eisenmenger-Sittner, Surf. Coat. Technol. 236, 353 (2013)

22. H. Kersten, H. Deutsch, G.M.W. Kroesen, Int. J. Mass Spectrom. 233, 51 (2004)

23. H. Kersten, H. Deutsch, E. Stoffels, W.W. Stoffels, G.M.W. Kroesen, Int. J. Mass Spectrom. 223-224, 313 (2003)

24. Sympatec GmbH System-Partikel-Techni, https://www. sympatec.com/DE/LaserDiffraction/HELOS.html

25. Refractive Index. INFO - Refractive index database, http://refractiveindex.info/

26. Win X-Ray - Electron Beam Scattering Modeling, http://montecarlomodeling.mcgill.ca/software/ winxray/winxray.html

27. Ye.P. Mamunya, H. Zois, L. Apekis, E.V. Lebedev, Powder Technol. 140, 49 (2004)

28. J. Sánchez-González, A. Macías-García, M.F. AlexandreFranco, V. Gómez-Serrano, Carbon 43, 741 (2005)

Open Access This is an open access article distributed under the terms of the Creative Commons Attribution License (http://creativecommons.org/licenses/by/4.0), which permits unrestricted use, distribution, and reproduction in any medium, provided the original work is properly cited. 